\title{
Chaos and Lyapunov exponents in classical and quantal distribution dynamics
}

\author{
Arjendu K. Pattanayak and Paul Brumer \\ Chemical Physics Theory Group, University of Toronto, Toronto, Ontario, Canada M5S 3 H6
}

(Received 24 July 1996; revised manuscript received 23 April 1997)

\begin{abstract}
We analytically establish the role of a spectrum of Lyapunov exponents in the evolution of phase-space distributions $\rho(p, q)$. Of particular interest is $\lambda_{2}$, an exponent that quantifies the rate at which chaotically evolving distributions acquire structure at increasingly smaller scales and is generally larger than maximal Lyapunov exponent $\lambda$ for trajectories. The approach is trajectory independent and is therefore applicable to both classical and quantum mechanics. In the latter case we show that the $\hbar \rightarrow 0$ limit yields the classical, fully chaotic, result for the quantum cat map. [S1063-651X(97)00111-6]
\end{abstract}

PACS number(s): 05.45.+b, 03.65.Sq

The canonical measure of chaotic Hamiltonian dynamics is the nonzero Lyapunov exponent [1], which quantifies the rate of exponential divergence of neighboring classical trajectories. This measure has resisted translation to the Hilbert space of phase-space distributions, where the linearity of the evolution equation precludes the asymptotic exponential divergence of initially "close" distributions. Rather, chaos in distributions is described in terms of the character of the spectrum of the Liouville operator [2], a difficult property to access. As a consequence, a quantitative diagnostic for the manifestation and characterization of chaos in the evolution of distributions has proven elusive, although several conjectures and limited diagnostics have been proposed [3-7].

In this article we analytically establish a diagnostic of chaos for distributions that is independent of trajectories and is valid for arbitrary initial distributions and at asymptotic times. In particular, we expose the role of an entire spectrum of generalized Lyapunov exponents $\lambda_{\gamma}$ [8] in the evolution of distributions. Although we derive the results by considering phase-space trajectories, our final expressions for $\lambda_{\gamma}$ depend solely on the evolution of the phase-space distribution. We thus have a means of studying the role of Lyapunov exponents in the evolution of both classical and quantum systems, as shown below.

We also show that the particular exponent $\lambda_{2}$, which is generally larger than the standard trajectory-based maximum Lyapunov exponent $\lambda[8]$, assumes particular importance as the arbiter of the growth of phase-space structure. This growth has been thought [9] to characterize chaos for distributions. In particular, we show that (i) a distribution evolves so as to acquire structure at increasingly smaller scales at an exponential rate given by $\lambda_{2}$, (ii) the rate at which the information in the system moves to the smaller scales (and hence the rate at which it relaxes) is given by $\lambda_{2}$ and (iii) the error in a calculation for a fixed level of resolution increases exponentially in time with $\lambda_{2}$.

Consider the usual computation of the Lyapunov exponents [1]: Let the equations of motion of a point in phase space be $\dot{x}_{i}=f_{i}(x)$, where $x[\equiv(q, p)$ for Hamiltonian systems] is the vector denoting all phase-space variables and individual vector components are denoted by subscripts. Our variables are scaled to be dimensionless so that we may define a metric in phase space. The equations of motion for the vectors in the tangent space are obtained by linearizing the equations of motion [substituting $x+s$ into $\dot{x}_{i}=f_{i}(x)$ ] around this fiducial trajectory:

$$
\frac{d \varsigma_{i}}{d t}=\sum_{j} M_{i j} \varsigma_{j}
$$

where $M_{i j}=\partial f_{j} / \partial x_{i}$ is the Jacobian matrix at the point $x(t)$. If this system is evolved forward in time, then we can obtain the maximal Lyapunov exponent as (theorems A and B, Ref. [1])

$$
\lim _{t \rightarrow \infty} \frac{1}{t} \ln [|\varsigma(t)|]=\lambda(x)
$$

where || defines the standard norm in phase space and the result is independent of $s$ except for a set of vanishing measure. For a Hamiltonian system the set of exponents is unchanged under the transformation $\lambda \rightarrow-\lambda$ as a consequence of time-reflection symmetry.

We use the foregoing to derive the equations of evolution for the first-order derivatives of a distribution. The assumptions necessary in our analysis imply that the results are valid for all distributions that evolve nontrivially under the Liouville equation and are differentiable: This specifically excludes $\delta$-function distributions (point trajectories) and timeinvariant solutions to Liouville's equation. Consider the relationship between the densities at the point $x$ and $x+\mathrm{s}$ :

$$
\rho(x+\varsigma)=\rho(x)+\sum_{i} \varsigma_{i} \frac{\partial}{\partial x_{i}} \rho(x),
$$

where we neglect higher-order terms, as in the trajectorybased derivation. Taking the total time derivative of both sides and noting that $d \rho(x) / d t \equiv 0 \equiv d \rho(x+s) / d t$, we get

$$
\sum_{i} \frac{d}{d t}\left[\varsigma_{i}\left(\frac{\partial \rho}{\partial x_{i}}\right)\right]=\sum_{i}\left[\frac{d \varsigma_{i}}{d t} \frac{\partial \rho}{\partial x_{i}}+\varsigma_{i} \frac{d}{d t}\left(\frac{\partial \rho}{\partial x_{i}}\right)\right]=0,
$$

where here and below $\rho$ and its derivatives are evaluated at $x$. Using Eq. (1), we get

$$
\begin{aligned}
\sum_{i} & {\left[\sum_{j} M_{i j} \varsigma_{j}\left(\frac{\partial \rho}{\partial x_{i}}\right)\right]+\sum_{j}\left[\varsigma_{j} \frac{d}{d t}\left(\frac{\partial \rho}{\partial x_{j}}\right)\right] } \\
& =0
\end{aligned}
$$




$$
\begin{aligned}
& \Rightarrow \sum_{j} \varsigma_{j}\left(\sum_{i}\left[M_{i j}\left(\frac{\partial \rho}{\partial x_{i}}\right)\right]+\frac{d}{d t}\left(\frac{\partial \rho}{\partial x_{j}}\right)\right\}=0 \\
& \Rightarrow \frac{d}{d t}\left(\frac{\partial \rho}{\partial x_{j}}\right)=-\sum_{i} M_{i j}\left(\frac{\partial \rho}{\partial x_{i}}\right)
\end{aligned}
$$

the last equality following from the independence of the various $\varsigma_{j}$. Equations (1) and (7) for the evolution of $\varsigma$ and $\nabla \rho$, respectively, are identical except for a minus sign. This makes physical sense: Since the number of points cannot be created or destroyed along trajectories, the density sharpens (flattens) along the direction in which points move closer (further). This being the case, $\lambda$ is directly reflected in the gradient of the distribution $\nabla \rho(x(t))$ at asymptotic times at almost every phase-space point [10] in the chaotic region. Remembering the reflection symmetry of the Lyapunov exponents, the maximal Lyapunov exponent may hence be equally well computed as

$$
\lambda(x)=\lim _{t \rightarrow \infty} \frac{1}{t} \ln [|\nabla \rho(x(t))|] .
$$

We now consider the averaged quantities $\chi_{\gamma}$ where

$$
\chi_{\gamma} \equiv\left(\frac{\operatorname{Tr}\left[|\nabla \rho(x(t))|^{\gamma}\right]}{4 \pi^{2} \operatorname{Tr}\left[\rho^{\gamma}(x(t))\right]}\right)^{1 / \gamma}
$$

Here $\operatorname{Tr}$ indicates the trace or integration over phase space and $\gamma$ is an arbitrary real number; note that the denominator $\operatorname{Tr}\left[\rho^{\gamma}(x(t))\right]$ is a constant for Hamiltonian evolution. Several interesting properties have been established for similarly constructed averages using $s$ instead of $\nabla \rho[8]$. Using the same approach, since Eqs. (2) and (8) are identical, we get that the following properties also hold for $\lambda_{\gamma}$ defined via Eq. (9): (i) A spectrum of generalized Lyapunov exponents can be defined through

$$
\lim _{t \rightarrow \infty} \frac{1}{t} \ln \left(\chi_{\gamma}\right)=\lambda_{\gamma}
$$

(ii) The "usual" maximal Lyapunov exponent $\lambda$ is a member of the spectrum $\lim _{\gamma \rightarrow 0} \lambda_{\gamma}=\lambda$, and (iii) $\lambda_{\gamma}$ is concave in $\gamma$, i.e., if $\gamma_{1}<\gamma_{2}$, then $\lambda_{\gamma_{1}} \leqslant \lambda_{\gamma_{2}}$. Note that by defining the averages in terms of $\nabla \rho$ rather than s, the quantities $\chi_{\gamma}$ and $\lambda_{\gamma}$ are independent of trajectories.

We note that the dependence of $\lambda_{\gamma}$ on $\gamma$ arises due to the variation in local stretching rates in different regions of phase space. Hence, for a system where the local stretching rate is a constant, $\lambda_{\gamma}$ is independent of $\gamma$ and equals the maximum Lyapunov exponent $\lambda$.

Considerable physical insight emerges by considering $\lambda_{2}$ and the quantity $\chi_{2}(t)$ in Fourier space. That is, consider the Fourier expansion of a distribution $\rho(q, p)$ $=\sum_{n, m} \rho_{n, m}(t) f_{n, m}$, where $f_{n, m}(p, q)=\exp \{2 \pi i(n p+m q)\}$. We use a two-dimensional discrete Fourier basis for simplicity; all arguments generalize to multiple dimensions and Fourier integrals. In Fourier space $\chi_{2}(t)$ is of the form

$$
\chi_{2}^{2}(t) \equiv \frac{\operatorname{Tr}\left[|\nabla \rho(x(t))|^{2}\right]}{4 \pi^{2} \operatorname{Tr}\left[\rho^{2}(x(t))\right]}=\frac{\sum_{n, m}\left(n^{2}+m^{2}\right)\left|\rho_{n, m}(t)\right|^{2}}{\sum_{n, m}\left|\rho_{n, m}(t)\right|^{2}}
$$

Hence $\chi_{2}$ is the root-mean-square Fourier radius, measuring the Fourier-space extent of the phase-space distribution. Equation (10) shows that

$$
\lim _{t \rightarrow \infty} \frac{1}{t} \ln \left(\chi_{2}\right)=\lambda_{2}
$$

or that, for a chaotic system, $\chi_{2}$ increases exponentially with time. Thus, since the higher $(|n|,|m|)$ modes correspond to structure at smaller scales, a distribution moves exponentially in time, with rate $\lambda_{2}$, from structure at a discernible scale to structure at extremely small scales. We can relate this to the loss of accuracy associated with the chaotic evolution of point trajectories as follows [11]. All the information about the distribution is encoded in the initial Fourier basis expansion; as $\chi_{2}$ grows, this information leaves any finite range of $|n|,|m|$ exponentially fast. Attempting to account for all Fourier modes corresponds to retaining an infinite amount of information and is inconsistent with a finiteresolution measurement in phase space. Thus Fourier modes with mode numbers greater than some $n_{\max }, m_{\max }$, where $1 / n_{\max }, 1 / m_{\max }$ are the limits of resolution in $p, q$ respectively, are effectively nonobservable and the information therein not retrievable. In this sense the evolution of a chaotic distribution can be reconciled with the exponential loss of accuracy inherent in the chaotic evolution of trajectories, also a consequence of finite measurement capability.

Equation (10) provides deep insights into the relation between the Lyapunov exponents and distribution dynamics but should not be regarded as a competitive tool to compute Lyapunov exponents when trajectories can be calculated. However, since $\chi_{\gamma}(t)$ no longer relies on trajectories, we may now study it for quantum mechanical phase-space distributions, thus analyzing "quantum chaos," i.e., the effect of chaos on quantal evolution. Again, $\chi_{2}$ proves particularly valuable since, by integration by parts,

$$
\chi_{2}^{2}(t) \equiv \frac{\operatorname{Tr}\left[|\nabla \rho|^{2}\right]}{4 \pi^{2} \operatorname{Tr}\left[\rho^{2}\right]}=-\frac{\operatorname{Tr}\left[\rho \nabla^{2} \rho\right]}{4 \pi^{2} \operatorname{Tr}\left[\rho^{2}\right]}
$$

Noting that $\nabla^{2} \rho \equiv\{p,\{p, \rho\}\}+\{q,\{q, \rho\}\}$, where $\{$,$\} denotes$ the Poisson bracket, we may now perform the standard quantization $\{A, B\} \rightarrow-(i / \hbar)[\hat{A}, \hat{B}]$ to yield a quantal $\chi_{2}$ that is independent of the representation. In the Wigner-Weyl [13] representation it has precisely the classical form [Eq. (13)], with $\rho$ replaced by the Wigner function $W(q, p)$.

As an example of the insights afforded by this approach we consider the cat map on the torus, which is a classical $K$ system [12] and has been shown recently [7] to display smooth quantum-classical correspondence. The dynamics of this system, whose classical and quantal propagators for distributions are known analytically [14], are those of a kicked oscillator with Hamiltonian [11] 


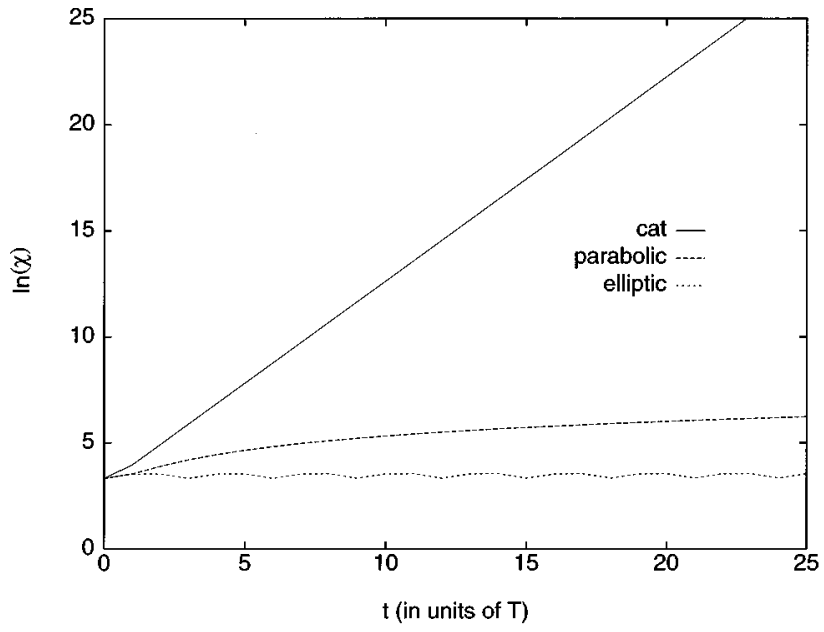

FIG. 1. $\chi$ as a function of time for the classical elliptic, parabolic, and cat maps.

$$
H=p^{2} / 2 \mu+\epsilon q^{2} / 2 \sum_{s=-\infty}^{\infty} \delta(s-t / T),
$$

restricted to a torus $0 \leqslant q<a, 0 \leqslant p<b$. The cat map corresponds to the choice $\eta=T b / \mu a=1$ and $\xi=-\epsilon T a / b=1$ and $\alpha=h / a b$ acts as a dimensionless form of Planck's constant for this problem. Two other cases of interest we study are the (stable) elliptic map $(\eta=1, \xi=-1)$ and the borderline parabolic case $(\eta=1, \xi=0)$. We note that this classical system has a constant Jacobian matrix and $\chi_{\gamma} \equiv \chi$ is independent of $\gamma$.

We then anticipate that (i) for the stable system $\chi$ oscillates as a function of time, (ii) $\chi$ grows linearly for the parabolic case, and (iii) a chaotic system has $\chi$ growing exponentially in time with the asymptotic rate given by $\lambda$. This behavior is indeed confirmed computationally, as shown in Fig. 1, for these three maps. We emphasize that (i) the behavior is independent of the initial distribution (the distributions used here were randomly initialized) and (ii) in the chaotic case, there is no saturation of $\chi(t)$ even though the phase-space is bounded.

In the quantal case we study the dependence of $\chi_{2}(t)$ on the degree of classicality of the system, that is, the value of $\alpha$. To eliminate kinematical effects of changing $\alpha$ we consider the evolution of Wigner distributions that are initially of the Gaussian form

$$
W(q, p)=N_{\beta} \exp \left[-\frac{\left(q-\beta q_{0}\right)^{2}}{\beta^{2} \sigma_{q}^{2}}\right] \exp \left[-\frac{\left(p-\beta p_{0}\right)^{2}}{\beta^{2} \sigma_{p}^{2}}\right]
$$

where $\beta$ is a scaling variable (see below), $N_{\beta}$ is a normalization factor, and $\left(\beta q_{0}, \beta p_{0}\right)$ specifies the location of the Gaussian of width $\left(\beta \sigma_{q}, \beta \sigma_{p}\right)$ in a phase space of dimension $\beta^{2} a b$. Since $\alpha=h / \beta^{2} a b$, by increasing $\beta$ we approach the classical limit while preserving the ratio $\left(\beta^{2} \sigma_{q} \sigma_{p} / \beta^{2} a b\right)$ of the volume of the initial distribution to the volume of phase space. Here we choose this ratio as $\approx 0.0175$, sufficiently large to display quantum effects at relatively early times. Note that for these large initial distri-

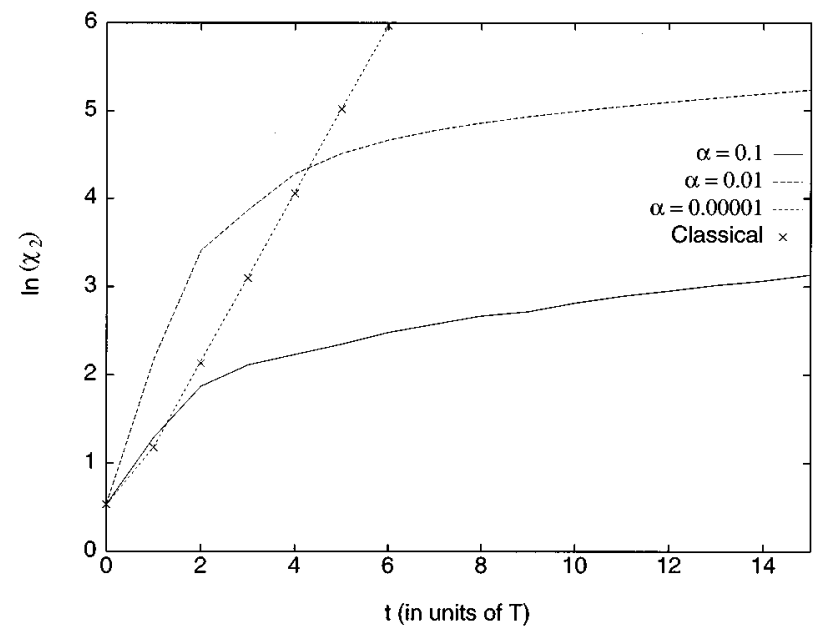

FIG. 2. $\chi_{2}(t)$ for the classical $(\alpha=0)$ and quantal $\left(\alpha=10^{-1}\right.$, $10^{-2}$, and $10^{-5}$ ) cat map.

butions, the approximate methods of measuring the separation of centroids [7] or the growth of second moments [6] fail to detect the existence of chaos, even for the classical system. Our results are displayed in Fig. 2.

For the classical cat $(h=0, \beta a=\beta b=1)$, shown again for comparison, $\chi_{2}(t)$ grows exponentially after a short transient, with a rate equal to $\lambda$. The behavior of $\chi_{2}$ for the quantized cat map is shown for $\alpha=10^{-5}, 10^{-2}$, and $10^{-1}$. Near the classical limit $\left(\alpha=10^{-5}, h=1, \beta a=\beta b=316.2\right)$ $\chi_{2}$ exhibits exponential growth, indistinguishable from the classical behavior on the finite Fourier grid (of size $2048 \times 2048$ ) used in our computations. However, as $\alpha$ is increased to $10^{-2}(h=1, \beta a=\beta b=10)$ the initial growth of $\chi_{2}$ is seen to be faster than that of the classical cat map, suggesting that the amount of structure in the quantal distribution at short times actually increases relative to the classical one. This is indeed the case since one expects the quantal $W(q, p)$ to adiabatically follow the classical $\rho(q, p)$, but with added fringes [15] (interference structures). Such interference structures can be seen in contour maps of the evolving distributions. For example, an examination of Figs. 1-3 of Ref. [7] at $t=4 T$ shows clearly that the distribution with $\alpha=10^{-2}$ has far more structure at finer scales than does the distribution with $\alpha=10^{-5}$. This, of course, implies greater support for the distribution at larger Fourier node numbers, leading to larger $\chi_{2}$ values. However, supraclassical growth does not persist for larger $\alpha$ or for longer time because a second quantal effect kicks in: Quantal distributions resist the growth of structure at scales smaller than $\hbar$ [15], implying that the support of the distribution travels slowly across the $1 / \alpha$ boundary in Fourier space. This is marked by a clear slowdown of the growth at $\chi_{2} \approx 100=O(1 / \alpha)$. As $\alpha$ is increased to $10^{-1}(h=1, \beta a=\beta b=3.162)$, the initial rapid growth saturates in one time step, resulting in a slowly growing $\chi_{2}$. The interplay between the two effects thus implies in general a finite value of $\alpha$ at which the quantum system acquires structure maximally rapidly for early times. Note also that the initial differences between the various values of $\chi_{2}$ in Fig. 2 is exaggerated by the fact that we are using discrete time steps, which are themselves of the order of $1 / \lambda_{2}$ in our model system. 
Since the quantal propagator is a smooth function of $\alpha$, the results at larger values of $\alpha$ imply that on the full (infinite) Fourier grid the quantal $\chi_{2}$, even at the smallest values of $\alpha$, will ultimately slow down, reflecting the nonergodic nature of the quantum map [14]. However, and this point is significant, for any finite resolution there is always an $\alpha$ sufficiently small such that the classical and quantal behavior are indistinguishably chaotic. Thus the various $\lambda_{\gamma}$ govern the behavior of classical and quantal distributions in precisely the same manner in the limit $\hbar \rightarrow 0$, as manifest in the behavior of $\chi_{2}$. Note that the form of $\chi_{2}(t)$ enables us to verify this behavior without masking effects arising from the choice of broad initial distributions or from saturation and relaxation in the finite phase space (see the discussion in Ref. [7]).

In summary, we have provided a quantitative demonstration of the role of Lyapunov exponents in the behavior of classical and quantal distributions and have identified $\chi_{2}(t)$ as a measure of the increasingly fine detailed structure of the distribution. We have thus related the generalized Lyapunov exponents directly to the properties of distributions, independent of trajectories. Our approach is valid for arbitrary initial distributions and asymptotic times, unlike other methods $[6,7]$, and has been explicitly derived from the definition of Lyapunov exponents, unlike previous conjectures [3-5]. In particular, it avoids the need to introduce perturbations to define the stability of distributions [3] and avoids basisdependent definitions of chaos in distributions [4]. Rather, it provides a consistent understanding of the role of Lyapunov exponents in the dynamics of classical distributions and affords a method of connecting Lyapunov exponents to the behavior of quantal distributions in the semiclassical limit $\hbar \rightarrow 0$.

Note added in proof. Recently we found that our work overlaps that in Y. Gu, Phys. Lett. A 149, 95 (1990).

This research was supported by the Natural Sciences and Engineering Research Council of Canada. A.K.P. would like to thank Salman Habib for useful discussions.
[1] G. Benettin, L. Galgani, and J.-M. Strelcyn, Phys. Rev. A 14, 2338 (1976).

[2] P. R. Halmos, Lectures on Ergodic Theory (Chelsea, New York, 1956).

[3] A. Peres, Phys. Rev. A 30, 1610 (1984); R. Schack and C. M. Caves, Phys. Rev. Lett. 71, 525 (1993), and references therein.

[4] A. Peres and D. Terno, Phys. Rev. E 53, 284 (1996); A. Peres, ibid. 53, 4525 (1996).

[5] L. E. Ballentine and J. P. Zibin, Phys. Rev. A 54, 3813 (1996).

[6] R. C. Fox and T. C. Elston, Phys. Rev. E 49, 3683 (1994), and references therein.

[7] A. K. Pattanayak and P. Brumer, Phys. Rev. Lett. 77, 59 (1996).

[8] C. Beck and F. Schlögl, Thermodynamics of Chaotic Systems (Cambridge University Press, New York, 1993); G. Paladin and A. Vulpiani, Phys. Rep. 156, 147 (1987); H. Fujisaka, Prog. Theor. Phys. 70, 1264 (1983).
[9] See, e.g., B. Chirikov, F. Izrailev, and D. Shepelyansky, Physica D 33, 77 (1988).

[10] We say "almost every" to exclude unstable periodic orbits, a set of zero measure.

[11] J. Ford, G. Mantica, and G. H. Ristow, Physica D 50, 493 (1991), and references therein.

[12] V. I. Arnold and A. Avez, Ergodic Problems of Classical Mechanics (Addison-Wesley, New York 1989).

[13] See S. R. De Groot and L. G. Suttorp, Foundations of Electrodynamics (North-Holland, Amsterdam, 1972) for an excellent review of the Wigner-Weyl technique.

[14] J. Wilkie and P. Brumer, Phys. Rev. E 49, 1968 (1994); J. Wilkie, Ph.D. dissertation, University of Toronto, 1994 (unpublished).

[15] H. J. Korsch and M. V. Berry, Physica D 3, 627 (1981), and in many papers by other authors since. 